\title{
Verbal interference with perceptual classification: The effect of semantic structure*
}

\author{
HOWARD HOCK $\div$ \\ Florida Atlantic L'niversity. Boca Raton. Florida 33432 \\ and \\ JACQUES PETRASEK \\ The Johns Hopkins Lini ersity, Baltimore. Marnland 21218
}

\begin{abstract}
In a task of the same form as the standard Stroop test. the relevant attribute was ellipse size and the required responses were the numbers 1 thirough 6 assigned to each of the ellipses in order of increasing size. The irreievant attribute consisted of either alphabet letters or the numerical symbols 1 through 6 displayed in the center of each ellipse. The numerals produced more interference with the classification of the relevant attribute than the alphabet let ters. supporting Klein's (1964) results. In addition, the interference due to the irrelevant numerical symbols increased as the distance between the values of the relevant and irrelevant attributes was decreased. Since "distance" is a structural property of the number system, this indicated that the competing response tendencies aroused by the irrelevant numericals involved the semantic structure for numbers. The same results were obtained when numerical quantity. rather than ellipse size, was the relevant attribute.
\end{abstract}

In 1935. Stroop performed a renowned experiment in which $\mathrm{Ss}$ classified the ink colors of incongruous color-word stimuli (color names conflicting with the ink color in which they were printed, e.g., the word "blue" printed in red ink). Slower response times for the incongruous color words, relative to control stimuli consisting of color patches, was explained in terms of the competing response tendencies aroused by the words. which the Ss were trving to ignore. In a more recent study. Klein (1964) found that the amount of interference depended on the semantic relation between the ink colors and the words. Using four classes of words-(1) words that were not directly related to the ink colors (e.g., put, friend). (2) words associatively connected to the ink colors (e.g.. grass, sky), (3) color words other than those used for the ink colors, and (4) color words conflicting with the responses to the ink colors (the standard Stroop condition)-Klein found that each word class provided successively more interference with the classification of the ink colors. The interference was attributed to competing response tendencies aroused by the irrelevant words via associative networks involving the semantic structure of the words and ink colors.

Although he clearly demonstrated that the amount of interference depended on the degree of semantic relation between the word class and the ink colors, Klein did not

*This experiment was supported in part by a contract between the Engineering Psychology Programs, Office of Naval Research. and the Johns Hopkins University, and in part by Florida Atlantic University Seed Grant 7035. The advice of Howard Egeth in all phases of this study is gratefully acknowledged. The authors would also like to thank Leon Gold, who collected the data for Experiment 2.

$\div$ Requests for reprints should be sent to Howard S. Hock, Department of Psychology: Horida Atlantic Liniversity. Boca Raton. llorida 33432. investigate the nature of the semantic relation within any of the word classes. That is, he did not indicate which, if any, semantic structures were involved in the semantic relation between the ink colors and any single class of interfering words.

One way of providing direct evidence that verbal interference with the classification of the relevant attribute rests upon a semantic structure is to show that a property of that structure affects the amount of interference. Since Deese (1965, p. 135) has pointed out that the semantic structure employed for color names is not easily specified. another perceptual domain was selected for this purpose. Ellipse size was chosen as the relevant attribute. and the required responses were the numbers 1 through 6 assigned to each of the ellipses in order of increasing size. The irrelevant attribute consisted of the numerical symbols 1 through 6 displayed in the center of each ellipse. It was hypothesized that the amount of interference in classifying an ellipse would depend on the numerical distance between the correct response to the ellipse and the irrelevant numeral in its center: the smaller the distance, the greater the expected interference. Since "distance" is a structural property of the number system, this would indicate that the interference with the classification of the relevant attribute involved the semantic structure for numbers.

\section{EXPERIMENT I}

\section{Method}

\section{Stimuli}

Each stimulus consisted of the black outline of a $60-\mathrm{deg}$ eccentricity ellipse on the white background of a $4>5 \mathrm{in}$. card. The ellipse diameters were $5 / 8,7 / 8,9 / 8,11 / 8,13 / 8$. and $2 \mathrm{in}$. 
Table 1

The Irrelevant Symbols Associated With Each Ellipse Size for the Letter (L), Distant Numeral (DN), and Close Numeral (CN) Conditions of Experiment I

\begin{tabular}{cccc}
\hline \multirow{2}{*}{$\begin{array}{c}\text { Ellipse } \\
\text { Size }\end{array}$} & \multicolumn{3}{c}{ Condition } \\
\cline { 2 - 4 } & L & DN & CN \\
\hline 1 & E,G,L,S & $4,4,5,6$ & $2,2,3,3$ \\
2 & E,G,R,L & $5,5,5,6$ & $1,1,3,4$ \\
3 & E,G,R,L & $6,6,6,6$ & $2,2,4,4$ \\
4 & E,G,R,S & $1,1,1,1$ & $3,3,5,5$ \\
5 & E,G,R,B & $2,2,2,1$ & $3,4,6,6$ \\
6 & E,G,L,B & $3,3,2,1$ & $4,4,5,5$ \\
\hline
\end{tabular}

for the easy discrimination condition and $1,9 / 8,10 / 8,11 / 8$, $12 / 8$, and $14 / 8$ in. for the difficult discrimination condition. In both conditions, the last step in the series of ellipse sizes was greater than the differences in size between the smaller ellipses so that the confusability of the larger ellipses would be consistent with that of the smaller ellipses.

In addition to discriminability, the stimuli varied with respect to the symbolic information in the center of the ellipse. In Condition L (letters), the irrelevant symbols were alphabet letters $(E, G, L, S, R$, and $B$ ); in Condition DN (distant numerals), they were numerals (from 1 through 6) arranged so that there was a distance of three, four, or five between the number indicating the size of the ellipse and the irrelevant numeral; and in Condition CN (close numerals), they were the same numerals, but arranged so that the distance was one or two. Conditions DN and $\mathrm{CN}$, therefore, differed with respect to the numerical distance between the values of the relevant and irrelevant attributes.

\section{Design}

Six decks of stimulus cards were formed, one for each of the six orthogonal combinations of discriminability (easy vs difficult) and the type of irrelevant symbol $(\mathrm{L}, \mathrm{DN}$, and $\mathrm{CN})$. There were 24 cards in each deck, four for each of the six ellipse sizes. The pairings of the ellipse sizes and the irrelevant symbols, which were the same in both discriminability conditions, are presented in Table 1.

Although each irrelevant symbol did not occur equiprobably the uncertainty of the irrelevant information was the same (2.44 bits) in the $\mathrm{L}, \mathrm{DN}$, and $\mathrm{CN}$ conditions. It can be seen from Table 1, however, that the relevant and irrelevant attributes were partially correlated. In Condition DN, for example, when the irrelevant symbol was a 1 , the possible values of the relevant attribute were reduced to the three largest ellipses, with Ellipse Size 4 being the most likely. This partial correlation is quantifiable as conditional uncertainty, or the amount of uncertainty in one attribute when the value of the second attribute is known (Garner, 1962, p. 57). The conditional uncertainties were $2.12,0.88$, and 1.22 bits for Conditions $L$, $\mathrm{DN}$, and $\mathrm{CN}$, respectively. This uncontrolled difference between the conditions was the unavoidable constraint resulting from varying the numerical distance between the values of the relevant and irrelevant attributes, and equalizing the amount of irrelevant information in each condition. It will be demonstrated in Experiment Il that these differences in conditional uncertainty did not influence the outcome of the present experiment.

A total of 36 Ss were divided into two groups; one group was assigned to the easy and the other to the difficult discrimination condition. Within each group, each $S$ was assigned to one of the six counterbalanced orders of the alphabet letter (L), distant numerals (DN), and close numerals (CN) conditions. Each $S$ worked on each of these conditions twice, with the order of conditions reversed the second time through. One possible order of conditions, for example, was $L, C N, D N, D N, C N, L$. The sequence of cards remained fixed within each deck, the $S$ working through the sequence in both the forward and reversed directions.

\section{Procedure}

The first part of the experiment took the form of a paired-associate learning task. Ss learned to associate the numbers 1 through 6 with each of the six ellipses; 1 was assigned to the smallest and 6 to the largest ellipse. Corrective feedback was provided after each response. The training was provided with one of two decks of cards, one for the easy and the other for the difficult discrimination condition. There were 18 cards in each deck, with each ellipse size appearing three times and an " $x$ " placed in the center of each ellipse. There were six paired-association trials, with a trial defined as once through the deck of 18 cards. By the last trial, the average error rate was under $7 \%$.

The Ss were then given practice at the actual experimental task, once again using the training deck. The deck of cards was placed face down in front of the Ss, who were required to: (1) turn the cards over, one at a time, (2) indicate aloud the identity of the ellipse, and (3) place the card face down into a $41 / 2 \times 6$ in. box. Ss were instructed to work as quickly as possible and told that accuracy was insufficient without speed. They were told to guess rather than linger on a stimulus for which they were unsure of the correct response. There were three practice trials using this procedure.

The same instructions and procedure applied to the experimental trials that followed. For each deck, the Ss were told beforehand whether there were alphabet letters or numerals in the center of the ellipses, but they were instructed to ignore this irrelevant information. Responses were recorded, as were the times for each trial (once through the deck of 24 cards).

\section{Subjects}

The Ss, 36 undergraduate male students at Johns Hopkins University, were paid $\$ 1.50$ for participating in the experiment. The session lasted $30 \mathrm{~min}$, including the training period.

\section{Results}

The data for the experimental conditions were analyzed for the following dependent measures: (1) T, the total response time per trial (once through the deck of 24 cards), (2) $\mathrm{H}_{\mathrm{T}}$, the amount of information transmitted per trial, and (3) $\mathrm{H}_{\mathrm{T}} / \mathrm{T}$, the rate of information transmission (see Garner, 1962). The total response time $(T)$, averaged over all experimental conditions, was $35.6 \mathrm{sec}$, or $1.5 \mathrm{sec} / \mathrm{stimulus}$ card. $\mathrm{H}_{\mathrm{T}}$, again averaged over all the experimental conditions, was 1.81 bits (the maximum amount of transmittable stimulus information was $2.59 \mathrm{bits} /$ trial). The data for $\mathrm{H}_{\mathrm{T}} / \mathrm{T}$ are presented in Fig. 1. This measure was emphasized since it tends to reduce $S$ variability by compensating for individual differences in trading off speed and accuracy. The same relations between the experimental conditions shown in the figure were also obtained when $\mathrm{H}_{\mathrm{T}}$ and $\mathrm{T}$ were considered separately. The results of analyses of variance for the three measures differed only with respect to the discriminability variable; which fell short of significance (at the 0.10 level) when the $T$ measure was used, and practice, which was significant only when $\mathrm{H}_{\mathrm{T}} / \mathrm{T}$ was the dependent measure. 
It can be seen from Fig. 1 that the type of irrelevant symbolic information (alphabet letters, "distant" numerats. and "close" numerals) affected the rate of information transmission: this effect was statistically significant $[F(2.48)=16.96 . p<.005]$. The difference between the easy and difficult discrimination conditions was also significant $[F(1.24)=8.16 . p<.01]$. but the interaction between discriminability and the kind of irrelevant symbolic information was not significant $[F(2.48)<1.0]$. Although the practice effect due to working on each condition twice was significant $[F(1.24)=5.98, p<.025]$. practice did not interact with the kind of irrelevant symbolic information $[F(2.48)=1.53, p>.10]$ or discriminabiilty $[F(1,24)=$ 3.08. $\mathrm{p}>$.05].

Planned orthogonal comparisons were performed on the difference between the irrelevant alphabet letters and the irrelevant numerals ( $\mathrm{L}$ vs $\mathrm{DN}+\mathrm{CN}$ ), as well as the difference in interference due to the numerical distance between the values of the relevant and irrelevant attributes (DN vs $\mathrm{CN}$ ). Using the $\mathrm{H}_{\mathrm{T}} / \mathrm{T}$ measure. the difference between irrelevant alphabet letters and numerals was found to be significant at the .02 level for both groups of Ss: $t(17)=2.76$ for the easy discrimination and $t(17)=2.77$ for the difficult discrimination. The difference between Conditions DN and $C N$ was also significant for both groups: $t(17)=$ 3.21. $p<.01$ for the easy discrimination and $t(17)=$ 2.65. $p<.02$ for the difficult discrimination. The same results were obtained when the time measure (T) was

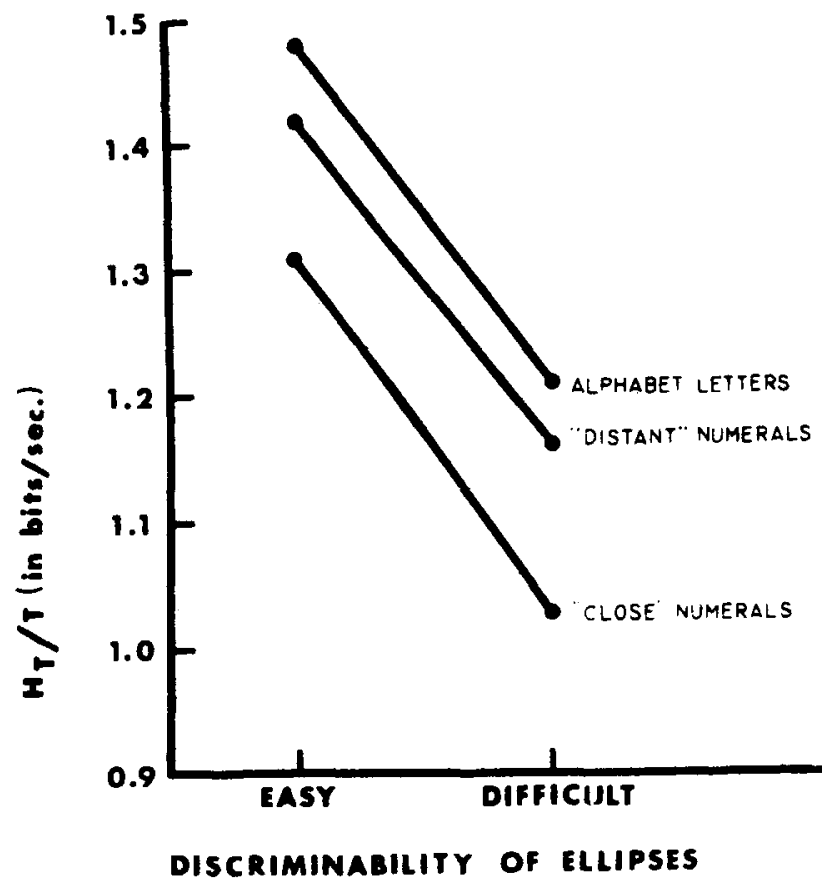

Fig. 1. The effect of irrelevant alphabet letters (L), "distant" numerals (DN), and "close" numerals (CN) on the rate of information transmission in classifying ellipse size in Experiment I.
Table 2

The Irrelevant Letters Associated With Each Ellipse Size for Conditions $L_{1}, L_{2}$, and $L_{3}$ of Experiment II

\begin{tabular}{|c|c|c|c|}
\hline \multirow{2}{*}{$\begin{array}{c}\text { Ellipse } \\
\text { Size }\end{array}$} & \multicolumn{3}{|c|}{ Condition } \\
\hline & $\mathrm{L}_{1}$ & $\mathrm{~L}_{2}$ & $\mathbf{L}_{3}$ \\
\hline 1 & E.G.L.S & G.G.L.S & B.B.E.E \\
\hline 2 & E.G.R.L & L.L.L.S & R.R.E.G \\
\hline 3 & E.G.R.L & S.S.S.S & B.B.G.G \\
\hline 4 & E.G.R.S & R,R.R.R & F,E,L.L \\
\hline 5 & E.G.R.B & $\mathrm{B}, \mathrm{B}, \mathrm{B}, \mathrm{R}$ & E.G.S,S \\
\hline 6 & E.G.L.B & E,E.B.R & $G . G, L, L$ \\
\hline
\end{tabular}

analyzed separately. When $\mathrm{H}_{\mathrm{T}}$ was the measure, however, all of the comparisons fell short of significance.

\section{EXPERIMENT II}

Although Conditions L. DN, and $\mathrm{CN}$ in Experiment I were intended to differ only with respect to the type of irrelevant symbolic information in the center of each ellipse, an unavoidable constraint in the design of the experiment resulted in $\mathrm{L}, \mathrm{DN}$, and $\mathrm{CN}$ also differing with respect to the amount of conditional uncertainty in the relationship between the relevant and irrelevant attributes. Although this made it possible for the Ss to use the value of the irrelevant attribute to help with the classification of the relevant attribute, there were several factors mitigating against this possibility: (1) the Ss were instructed to ignore the irrelevant symbols, (2) there is much evidence that attention to irrelevant verbal information produces interference with the classification of the relevant attribute, and (3) the amount of experience with the stimulus decks was insufficient for the Ss to have learned the complex mapping between the relevant and irrelevant attributes.

The purpose of this experiment was to provide empirical evidence that the differences in conditional uncertainty between Conditions $\mathrm{L}, \mathrm{DN}$, and $\mathrm{CN}$ could not have been responsible for the results obtained in Experiment 1.

\section{Method}

The only type of irrelevant symbols used in this experiment were alphabet letters. There were three experimental conditions $\left(L_{1}, L_{2}\right.$, and $L_{3}$ ) corresponding to Conditions $L . D N$, and $C N$ in Experiment $\mathrm{I}$. but with letters substituted for numerals in Conditions DN and CN. The pairings of letters and ellipse sizes. which are presented in Table 2. corresponded to the mapping between the relevant and irrelevant attributes in Experiment 1. As a result. Conditions $L_{1}, L_{2}$, and $L_{3}$ corresponded to Conditions L. DN. and CN of Experiment I with respect to the conditional uncertainty in the relationship between the relevant and irrelevant attributes. If differences in conditional uncertainty, and not the type of irrelevant symbolic information, were responsible for the results of Eiperiment I. significant differences between Conditions $\mathrm{L}_{1}, \mathrm{~L}_{2}$. and $\mathrm{L}_{3}$ would be obtained in the present experiment.

Since identical results were obtained in the casy and difficult discrimination conditions of 1 speriment $I$, only the former was used in this experiment. Otherwine. the experiment was procedurally identical to I periment l. Lighteen undergraduate 
and graduate students at Florida Atlantic University participated in this experiment, for which they were paid $\$ 1.75$.

\section{Results}

Using $\mathrm{H}_{\mathbf{T}} / \mathrm{T}$ as the dependent measure, mean information transmission rates of $1.38,1.41$, and 1.36 bits/sec were obtained for Conditions $L_{1}, L_{2}$, and $L_{3}$, respectively. The conditions did not differ significantly $\left[F(2,24)=0.90\right.$ for $\left.H_{T} / T\right]$, and the differences were similarly insignificant when $\mathrm{H}_{\mathrm{T}}$ and $\mathrm{T}$ were used as dependent measures.

It could be concluded, therefore, that the conditional uncertainty in the relationship between the relevant and irrelevant attribute was not responsible for the results of Experiment I.

\section{EXPERIMENT III}

The experiments of both Stroop and Klein were based on the over-learned relationship between the relevant attribute (ink color) and the required responses (color names). In Experiment I, however, the relationship between the relevant attribute (ellipse size) and the required responses (numbers) was based on a minimal amount of paired-associate learning. In order to demonstrate that the results of Experiment I were not a consequence of a weak stimulus-response relationship, an experiment of the same form was designed, but with a relevant attribute whose relationship to numerical responses was overlearned.

\section{Method}

The experiment was based on a procedure that has been used previously by both Windes (1968) and Morton (1969). The relevant attribute was the number or quantity of elements in each stimulus, with a range of values (1 through 6) within the limits of simultaneous apprehension or subitizing (Kaufman, Lord, Reese, \& Volkmann, 1949). The irrelevant attribute was the identity of the elements: they were either alphabet letters or numerals (from 1 to 6 ). The letter (L), distant numeral (DN), and close numeral (CN) conditions were identical to those of Experiment I, with values of the relevant and irrelevant attribute paired as in Table 1. In Condition $\mathrm{CN}$, for example, a response of "five" was required to the stimulus 44444 .

Unlike Experiment I, all the stimuli for each experimental condition were presented on the same sheet, with a trial defined as once through an entire sheet of 48 stimuli. The instructions were also altered: Ss were told to indicate the number of elements in each stimulus as quickly as possible, while minimizing their errors. Since errors were rare, only the total response time for each sheet was analyzed.

Twelve graduate students in psychology at Johns Hopkins University participated in this experiment, with each assigned to one of the six counterbalanced orders of the $L, D N$, and $C N$ conditions. As in Experiment l, each $S$ worked on each experimental condition twice, with the order of conditions reversed the second time through.

\section{Results}

The average response times per stimulus $(0.54 \mathrm{sec}$ for
Condition L, $0.57 \mathrm{sec}$ for Condition DN, and $0.60 \mathrm{sec}$ for Condition $\mathrm{CN}$ ) were in agreement with the results of Experiment I. The type of irrelevant symbolic information was significant $[F(2,12)=12.25, p<.05]$, and planned orthogonal comparisons indicated that the irrelevant numerals produced significantly more interference than the irrelevant alphabet letters ( $\mathrm{L}$ vs $\mathrm{DN}$ $+\mathrm{CN})[\mathrm{t}(11)=6.33, \mathrm{p}<.001]$ and the close numerals produced significantly more interference than the distant numerals $(\mathrm{CN}$ vs DN) $[\mathrm{t}(11)=2.23, \mathrm{p}<.05]$.

\section{DISCUSSION}

The experiments reported in this paper were designed to have the same form as the standard Stroop test, but with ellipse size and numerical quantity replacing ink color as the relevant attribute and numerical symbols replacing color words as the irrelevant attribute. For both the Stroop test and the present study, the values of the irrelevant attribute conflicted with the required responses to the relevant attribute.

Evidence that the rate of information transmission was significantly better for the irrelevant letters than for the irrelevant numerals ( $\mathrm{L}$ vs $\mathrm{DN}+\mathrm{CN}$ ) supported Klein's (1964) finding that interference with the classification of the relevant attribute depends on the semantic relation between the relevant attribute and the class of verbal information constituting the irrelevant attribute. The significant difference obtained between Conditions CN (close numeral) and DN (distant numeral) went a step further. It demonstrated that within a single class of irrelevant symbolic information (numerals), the amount of interference depends on the numerical distance between the values of the relevant and irrelevant attributes. Since "distance" is a structural property of the number system, it could be concluded that the interference caused by the irrelevant numerals involved the semantic structure for numbers.

It could be argued that the obtained effect of "distance" on interference had nothing to do with semantic structure, that it was instead the result of the S having to decide between alternative responses for the relevant attribute (ellipse size). If, for example, the correct response to a stimulus was 3 and the $S$ was uncertain whether to say 3 or 4 , more interference would occur if the irrelevant numeral were a 4 (as in Condition $\mathrm{CN}$ ) than if it were a 6 (as in Condition DN). If this were the case, however, more interference would have been obtained when the relevant attribute was more difficult to discriminate, since this would tend to increase the S's indecision. The absence of a significant interaction between discriminability and the type of irrelevant symbolic information was evidence against this alternative interpretation. Further evidence against explaining the results of Experiment 1 in terms of the S's judgmental indecision was obtained in Experiment III, which yielded the same results as Experiment $I$, but in a subitizing task involving simultaneous apprehension 
rather than dimensional judgment.

The results of this study supported the hypothesis that the semantic structure for numbers was involved in the interference with classification produced by irrelevant numerical symbols. While this research was based on a speeded classification task, DeSoto and Bosley (1962) have demonstrated how semantic structures affect performance in a different type of task. They found that Ss' learning to link men's names with the words freshman, sophomore, junior, or senior made errors that reflected the linear semantic structure underlying these social categories. Semantic structures may therefore affect both the accuracy with which a set of responses is learned and the speed with which those responses can be produced under interfering conditions.

\section{REFERENCES}

Deese, J. The structure of associations in language and thought.
Baltimore: The Johns Hopkins Press, 1965.

DeSoto. C. G., \& Bosley. J. J. The cognitive structure of a social structure. Journal of Abnormal \& Social Psychology, 1962, 64, 303-307.

Garner, W. R. Uncertainty and structure as psychological concepts. New York: Wiley, 1962.

Kaufman, E. L., Lord, M. W., Reese, T. W., \& Volkmann, J. The discrimination of visual number. American Journal of Psychology, 1949, 62, 498-525.

Klein, G. S. Semantic power measured through the interference of words with color names. American Journal of Psychology, 1964. 77, 576-588.

Morton, J. Categories of interference: Verbal mediation and conflict in card sorting. British Journal of Psychology, 1969, $60,329-346$.

Stroop, J. R. Studies of interference in serial verbal reactions. Journal of Experimental Psychology, 1935, 18, 643-662.

Windes, J. D. Reaction time for numerical coding and naming of numerals. Journal of Experimental Psychology, 1968, 78, 318-322.

(Received for publication December 16, 1970; revision received August 7,1972 .) 